\title{
Chemical Composition of Cynara Cardunculus L. var. altilis Heads: The Impact of Harvesting Time
}

\author{
Filipa Mandim ${ }^{1,2} \mathbb{D}^{\mathbb{D}}$, Spyridon A. Petropoulos $3, * \mathbb{C}$, Ângela Fernandes ${ }^{1} \mathbb{1}$, \\ Celestino Santos-Buelga ${ }^{2}$ (D) Isabel C. F. R. Ferreira ${ }^{1}$ (D) and Lillian Barros ${ }^{1, *(D)}$ \\ 1 Mountain Research Center (CIMO), Polytechnic Institute of Bragança, Campus de Santa Apolónia, \\ 5300-253 Bragança, Portugal; filipamandim@ipb.pt (F.M.); afeitor@ipb.pt (Â.F.); iferreira@ipb.pt (I.C.F.R.F.) \\ 2 The Polyphenols Research Group (GIP-USAL), Faculty of pharmacy, University of Salamanca, Campus \\ Miguel de Umaamuno s/n, 37007 Salamanca, Spain; csb@usal.es \\ 3 Department of Agriculture, Crop Production and Rural Environment, University of Thessaly, N. Iona, \\ 38446 Volos, Greece \\ * Correspondence: spetropoulos@uth.gr (S.A.P.); lillian@ipb.pt (L.B.)
}

Received: 2 July 2020; Accepted: 23 July 2020; Published: 27 July 2020

check for updates

\begin{abstract}
Cardoon is a multi-purpose crop with several industrial applications, while the heads (capitula) are edible and commonly used in various dishes of the Mediterranean diet. Several reports in the literature study the chemical composition of the various plants parts (leaves, flower stalks, bracts, seeds) aiming to industrial applications of crop bio-waste, whereas for the heads, most of the studies are limited to the chemical composition and bioactive properties at the edible stage. In the present study, cardoon heads were collected at six different maturation stages and their chemical composition was evaluated in order to determine the effect of harvesting stage and examine the potential of alternative uses in the food and nutraceutical industries. Lipidic fraction and the content in fatty acids, tocopherols, organic acids, and free sugars were determined. Lipidic content decreases with the maturation process, while 22 fatty acids were detected in total, with palmitic, oleic, and linoleic acids being those with the highest abundance depending on harvesting time. In particular, immature heads have a higher abundance in saturated fatty acids (SFA), whereas the samples of mature heads were the richest in monounsaturated fatty acids (MUFA). The $\alpha$-tocopherol was the only isoform detected being present in higher amounts in sample Car B (619 $\mu \mathrm{g} / 100 \mathrm{~g} \mathrm{dw})$. Oxalic, quinic, malic, citric and fumaric acids were the detected organic acids, and the higher content was observed in sample Car E (15.7 g/100 g dw). The detected sugars were fructose, glucose, sucrose, trehalose and raffinose, while the highest content $(7.4 \mathrm{~g} / 100 \mathrm{~g} \mathrm{dw})$ was recorded in sample Car C. In conclusion, the maturation stage of cardoon heads influences their chemical composition and harvesting time could be a useful means to increase the quality and the added value of the final product by introducing this material in the food and nutraceutical industries.
\end{abstract}

Keywords: seasonal variation; fatty acids; free sugars; chemical composition; Cynara cardunculus L.; cardoon; organic acids

\section{Introduction}

Cynara cardunculus L., or commonly known as cardoon, belongs to the Asteraceae family which is one of the largest families of the plant kingdom with more than 2000 species. Cynara cardunculus comprises three botanical varieties, all native to the Mediterranean basin, the wild cardoon (var. sylvestris), the domesticated cardoon (var. altilis DC), and the globe artichoke (var. scolymus) [1,2]. This crop has been gaining attention due to the high biological and industrial potential that it has 
shown in several studies described in the literature [1-4]. Despite being present all over the world, countries like Spain, France, and Italy are responsible for almost $80 \%$ of its production worldwide [1].

Cardoon is a species highly resistant to the fluctuation of weather conditions with low precipitation and hot and dry summers, characteristic of the Mediterranean basin climate. Its high resistance against adverse conditions and weather extremities, together with its multifaceted applicability, favor its exploration and the multiple uses in different industrial applications [4,5]. The industrial applications of cardoon are diverse, since it is used as plant rennet in the food industry to produce cheeses of protected designation of origin (PDO) [6,7]; it is also used for the production of paper pulp, due to its high content in cellulose and hemicellulose [8,9], as well as for bioenergy and biomass production $[1,10,11]$.

Cardoon is also used in traditional medicine since ancient times due to its health-promoting benefits. This species is widely consumed as a result of its antidiabetic, antihemorrhodial, cardiotonic, choleretic, and lipid-lowering actions. Furthermore, several studies have demonstrated other health-promoting properties, such as antioxidant, anti-HIV, anti-inflammatory, cytotoxic, antifungal, and antibacterial properties [4,12-15]. Several studies suggested that the miscellaneous medicinal properties confirmed so far are related to the presence of a high variety of bioactive compounds and phytochemicals. For this purpose, cardoon tissues are being widely explored as a result of its high concentration and variety in compounds with important biological effects and industrial purposes $[4,5,16,17]$. Literature reports also indicate cardoon as an important source of dietary components such as fibers, inulin, and minerals, but also of phenolic acids, mostly caffeoylquinic and dicafeoylquinic acids derivatives, flavonoids such luteolin and apigenin derivatives, anthocyanins and sesquiterpene lactones [8,18-21]. The presence and abundance of the detected biological compounds could be influenced by several factors, namely by the genotype, the pre and post-harvest conditions, the parts considered for chemical analysis (heads, leaves, bracts, flowers, pappi, receptacle, and petioles), the growing conditions, and the physiological stage at harvest $[4,19,22-25]$, while the choice of harvesting time has been suggested to affect the polyphenols content and composition [20]. Although the chemical composition of cardoon is widely described in the literature, further studies are needed to evaluate the influence of the abovementioned parameters on its chemical composition, thus allowing a more complete knowledge and the adequate use of the species based on specific bioactive compounds content.

Considering the lack of information in the scientific literature, the aim of this study was to determine the impact of the maturation stage on the lipidic content and on the profile of fatty acids, tocopherols, organic acids, and free sugars present in cardoon heads collected in central Greece and to evaluate alternative uses of cardoon heads that are overripe, e.g., they have passed the edible stage. The results of this study will contribute to better understanding the influence of the seasonal changes on the chemical composition of cardoon, resulting in an in-depth knowledge of the species and possible alternative uses in different areas of application that would add economic importance in this multi-purpose crop.

\section{Materials and Methods}

\subsection{Standards and Reagents}

All solvents were of analytical grade and were purchased from Fisher Scientific (Lisbon, Portugal). The fatty acids methyl esters (FAME) mixture (standard 47885-U) and standards of organic acids and sugars were acquired from Sigma-Aldrich (St Louis, MO, USA). Tocopherol standards were acquired from Matreya (Pleasant Gap, PA, USA). Other reagents and solvents of analytical grade were purchased from common sources. Water treatment was performed using a Milli-Q water purification system (TGI Pure Water Systems, Greenville, SC, USA). 


\subsection{Plant Material}

Head (capitulum) samples of Cynara cardunculus var. altilis DC cv. Bianco Avorio (Fratelli Ingegnoli Spa, Milano, Italy) were collected from the experimental farm of the University of Thessaly in Velestino, in central Greece (22.756 E, 39.396 N), during the growing period of 2017-2018 (Figure 1). Heads were collected from 15 individual plants $(n=15)$ at the beginning of the flower's development, in full maturity, and in seed ripening stages. The climate conditions during the growing period and the procedure used for the collection and treatment of plant material were described by Mandim et al. [19]. Briefly, Car A corresponds to principal growth stage (PGS) 5 (harvest: 26 April 2018), Car B corresponds to PGS 5/6 (harvest: 10 May 2018), Car C corresponds to PGS 6 (harvest: 24 May 2018), Car D corresponds to PGS 6/7 (harvest: 04 July 2018), and finally the samples Car E and Car F correspond to PGS 7 and PGS8, respectively (harvest: 09 August 2018 and 29 August 2018, respectively) [19,26].
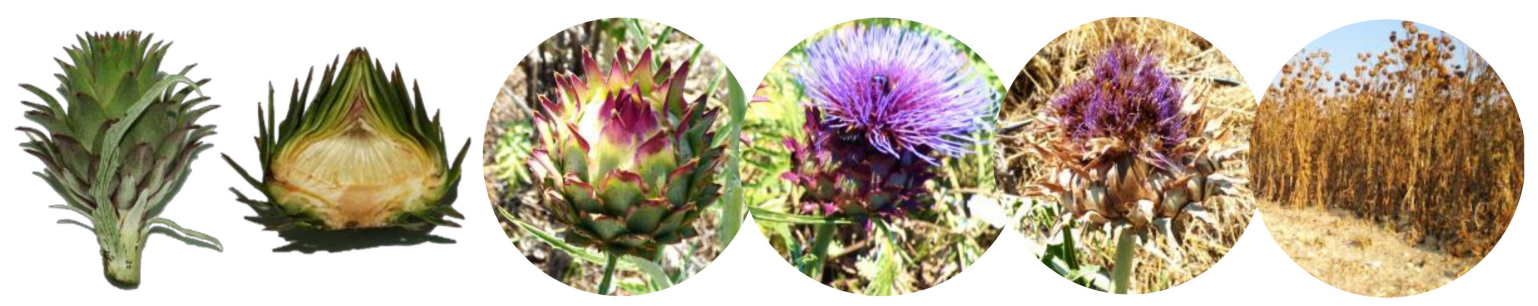

Maturation state

Figure 1. Evolution of maturity (Car A to F from left to right) of Cynara cardunculus L. heads during the growing period.

The plant material and the growing condition have been described in previous reports by our team. Briefly, bract samples were collected from 8-year-old plants sexually propagated from seeds in 2010. Soil parameters were the following, as previously described by the authors [27]: loam texture (48\% Sand; 29\% Silt; 23\% Clay); Organic matter: 1.3\%; pH: 7.9; EC: 1.4 dS/m; NO ${ }^{-3}: 9.49$ mg/kg; P: 74.53 mg/kg; $\mathrm{K}_{\text {exch }}: 0.98 \mathrm{cmol}_{\mathcal{C}} / \mathrm{kg} ; \mathrm{Ca}_{\text {exch }}: 13.96 \mathrm{cmol}_{\mathrm{C}} / \mathrm{kg} ; \mathrm{Mg}: 4.32 \mathrm{cmol}_{\mathrm{C}} / \mathrm{kg}$. Prior to crop establishment a base dressing was applied by using $50 \mathrm{~kg} / \mathrm{ha} \mathrm{N}, 90 \mathrm{~kg} / \mathrm{ha} \mathrm{P}_{2} \mathrm{O}_{5}$ and $40 \mathrm{~kg} / \mathrm{ha} \mathrm{K}_{2} \mathrm{O}$. After crop establishment, nitrogen fertilizers were applied with side dressing at each growing period and before plant regrowth (100 kg/ha N). Plant density was 40,000/ha with distances of approximately $0.6 \mathrm{~m}$ between rows and $0.4 \mathrm{~m}$ within rows. Irrigation was applied monthly during the first growing period (staring on April and until July) with water cannons, whereas in the following years irrigation was applied only twice in each growing period (on April and May) due to the extensive root system that plants form after the second year of establishment. Weed control was applied with hoeing after plant regrowth at each growing period, since at later growth stages plant is very competitive against weeds. No pesticides and fungicides were applied. Climate conditions during the experimental period (shoot emergence until senescence) are presented in Figure 2. 


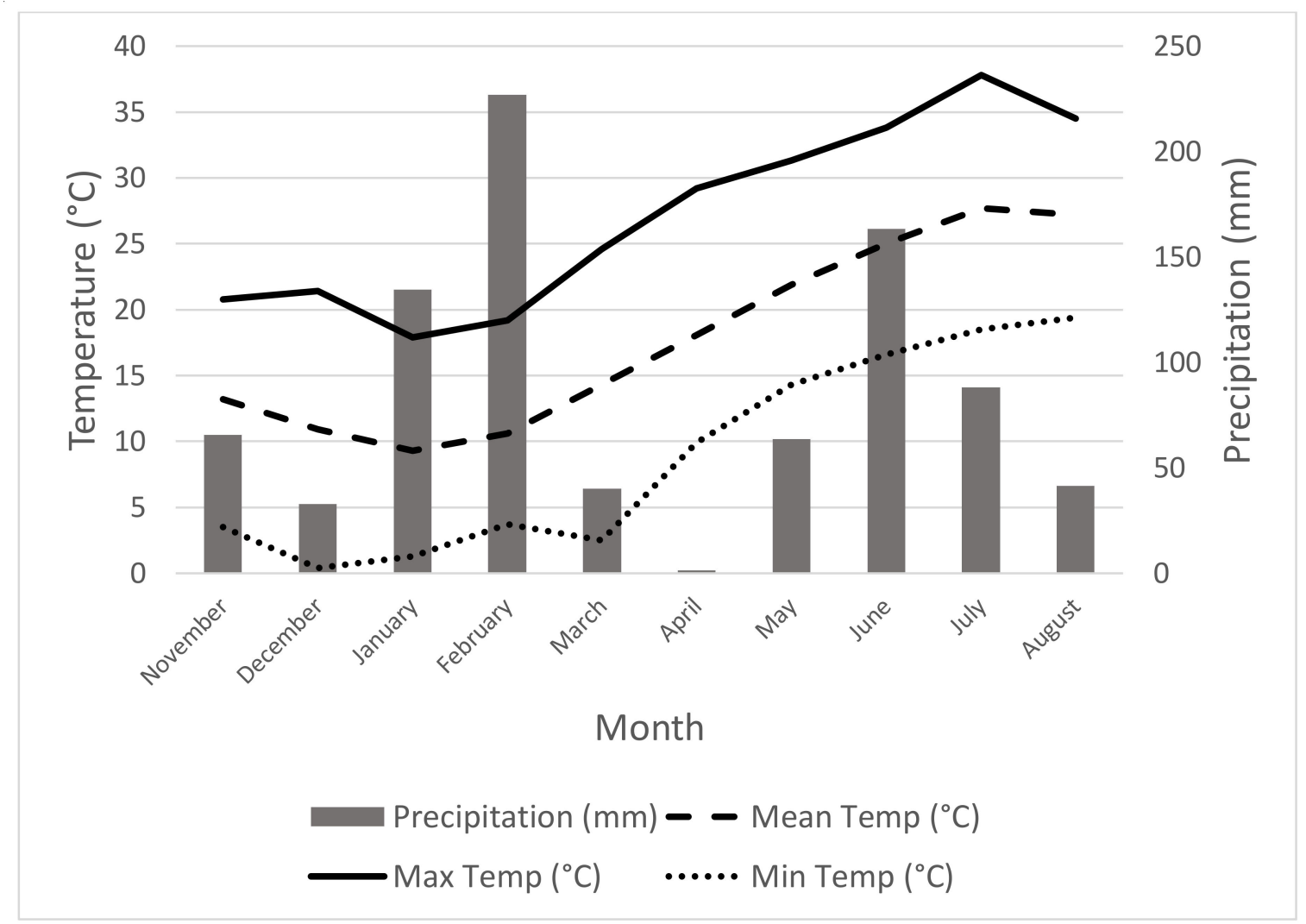

Figure 2. Climate conditions during the experimental period (Mean temperature $\left({ }^{\circ} \mathrm{C}\right)$; Max temperature $\left({ }^{\circ} \mathrm{C}\right)$; Min temperature $\left({ }^{\circ} \mathrm{C}\right)$; Precipitation $(\mathrm{mm})$.

\subsection{Chemical Composition Analysis}

\subsubsection{Fatty Acids}

For the analysis of fatty acids composition, the lipidic fraction was extracted with petroleum ether through a Soxhlet extraction system at $120^{\circ} \mathrm{C}$. After a transesterification process, the fatty acids content was analyzed by Gas-liquid Chromatography (GC), coupled to a Flame Ionization Detector (FID) at $260^{\circ} \mathrm{C}$ and according to the analytical conditions previously described [27]. The identification and quantification of fatty acids were performed by comparing the relative retention times of FAME peaks from samples with standards (reference standard mixture 47,885-U), using Clarity DataApex 4.0 software (Prague, Czech Republic). The results were expressed as relative percentages and in mg per $100 \mathrm{~g} \mathrm{dw}$ of each detected fatty acid.

\subsubsection{Tocopherols}

Tocopherols composition was analyzed using a high-performance liquid chromatography system (HPLC, Knauer, Smartline system 1000, Berlin, Germany) coupled to a fluorescence detector (FP-2020; Jasco, Easton, USA) programmed for excitation at $290 \mathrm{~nm}$ and emission at $330 \mathrm{~nm}$, according to the procedure previously described [28]. The qualitative and quantitative analysis were performed using the Clarity 2.4 software (DataApex, Prague, Czech Republic) and was achieved through comparison of the chromatographic data (retention times and spectra) with commercial standards, using the internal standard method. Tocopherols standards $(\alpha-, \beta-, \gamma-$, and $\delta$-isophorms, ) were used for compounds identification and quantification by the internal standard method. Results were expressed in $\mathrm{mg}$ per $100 \mathrm{~g}$ of dry weight $(\mathrm{dw})$ and were processed using the Clarity 2.4 software (DataApex, Prague, Czech Republic). 


\subsubsection{Organic Acids}

Organic acids were determined following the procedure previously described [29]. The samples were analyzed by Ultrafast Liquid Chromatography (UPLC, Shimadzu 20A series, Kyoto, Japan) coupled to a Diode Array Detector (UFLC-PDA, Shimadzu Corporation, Kyoto, Japan). Data were analyzed using the LabSolutions Multi LC-PDA software (Shimadzu Corporation, Kyoto, Japan). The identification was accomplished through the comparison of the retention times and the spectra obtained to the commercial standards (oxalic, quinic, malic, ascorbic, citric and fumaric acids), and their respective calibration curves were used to determine the quantity based on the area of the peaks. Results were expressed in g per $100 \mathrm{~g}$ of $\mathrm{dw}$.

\subsubsection{Free Sugars}

Free sugars content was analyzed by High Performance Liquid Chromatography (HPLC, Knauer, Smartline system 1000) coupled to a refractive index detector (RI detector, Knauer Smartilne 2300, Knauer, Berlin, Germany), according to the analytical conditions previously described [30]. Data was analyzed using the Clarity 2.4 software (DataApex) and the identification was performed through the comparison with standards (D (-)-fructose, D (+)-sucrose, D (+)-glucose, D (+)-trehalose and D (+)-raffinose pentahydrate (Sigma-Aldrich, St. Louis, MO, USA). The quantification was performed using melezitose (Matreya, PA, USA) as internal standard (IS). The results were processed through Clarity 2.4 software (DataApex, Prague, Czech Republic) through the comparison of retention times, UV-Vis and mass spectra of the sample compounds with those obtained from the available standards and the literature information available and presented in $\mathrm{g}$ per $100 \mathrm{~g}$ of dry weight $(\mathrm{dw})$.

\subsection{Statistical Analysis}

All the performed experiments were executed in triplicate. Results were presented as mean value \pm standard deviation. Means and standard deviations were calculated using Microsoft Excel. Differences among samples were analyzed using SPSS Statistics software (IBM SPSS Statistics for Mac OS, Version 26.0. Armonk, NY: IBM Corp.). The results were subject to an analysis of variance (ANOVA), while the Tukey's HSD test $(\alpha=0.05)$ was used to assess the significant differences between the samples. For the comparison between two samples, a two-tailed paired Student's $t$-test was applied to assess the statistical differences $(\alpha=0.05)$.

Moreover, a Principal Component Analysis (PCA) was performed in order to examine the contribution of each variable to the total diversity and classify the studied maturation stages according to their chemical composition and nutritional value by using the statistical software Statgraphics 5.1.plus (Statpoint Technologies, Inc., VA, USA).

\section{Results}

\subsection{Lipid Fraction and Fatty Acids Composition}

In Table 1 are presented the lipid and tocopherols content of cardoon heads collected at different maturation stages. The highest total lipidic fraction and a-tocopherol content was observed at early maturity stages (Car A and Car B for total lipidic fraction and $\alpha$-tocopherol content, respectively) and immature heads (Car A) had 10.9 times higher lipidic content than the sample of late maturity (Car F). In contrast, the progress of maturation process resulted in a gradual decrease of total lipid fraction, whereas $\alpha$-tocopherol content showed fluctuating trends with the lowest content being observed in Car C samples (mid-maturity stages). In particular, the highest amount was detected in the sample Car B (PSG 5/6) (619 $\mu \mathrm{g} / 100 \mathrm{~g} \mathrm{dw})$, while sample Car C (PSG 6) presented the lowest abundance $(25 \mu \mathrm{g} / 100 \mathrm{~g} \mathrm{dw})$. 
Table 1. Lipidic fraction and tocopherols of Cynara cardunculus heads at various stages of maturity (mean $\pm \mathrm{SD} ; \mathrm{n}=3$ ).

\begin{tabular}{ccc}
\hline Sample & Total Lipidic Fraction $\mathbf{( g / 1 0 0 ~} \mathbf{g ~ d w})$ & $\boldsymbol{\alpha}$-Tocopherol $(\boldsymbol{\mu g} / \mathbf{1 0 0} \mathbf{g ~ d w})$ \\
\hline Car A & $17.5 \pm 0.1^{\mathrm{a}}$ & $264 \pm 1^{\mathrm{b}}$ \\
Car B & $5.1 \pm 0.1^{\mathrm{b}}$ & $619 \pm 4^{\mathrm{a}}$ \\
Car C & $3.5 \pm 0.2^{\mathrm{d}}$ & $25 \pm 2^{\mathrm{f}}$ \\
Car D & $4.31 \pm 0.04^{\mathrm{c}}$ & $107 \pm 1^{\mathrm{e}}$ \\
Car E & $1.9 \pm 0.2^{\mathrm{e}}$ & $162 \pm 1^{\mathrm{c}}$ \\
Car F & $1.6 \pm 0.1^{\mathrm{f}}$ & $117 \pm 5^{\mathrm{d}}$ \\
\hline
\end{tabular}

Results are presented as mean \pm standard deviation. Different letters correspond to significant differences according to Tukey's HSD test $(p<0.05)$. dw-dry weight. Tocopherols calibration curves: $\alpha$-tocopherol $\left(y=1.295 x ; R^{2}=0.991\right.$; $\mathrm{LOD}=18.06 \mathrm{ng} / \mathrm{mL} ; \mathrm{LOQ}=60.20 \mathrm{ng} / \mathrm{mL})$.

Regarding the fatty acid composition of the tested cardoon heads in relation to maturity stage, the results are presented in Table 2, as well as the total saturated fatty acids, (SFA), monounsaturated fatty acids (MUFA), and polyunsaturated fatty acids (PUFA) content and PUFA/SFA and n-6/n-3 ratios. The values of the concentrations of all the referred parameters are provided as Supplementary Materials (Table S1). Thirty individual fatty acids were identified, with the palmitic (C16:0; 14.62-43.8\%), oleic (C18:1n9c; 4.48-46.6\%), and linoleic (C18:2n6c; 0.748-30.6\%) acids being detected in higher abundance. A typical chromatogram of fatty acids profile is presented in Figure 3. The highest content of palmitic acid was observed at early maturity stages (sample Car A), while a gradual decrease was observed as a maturation progress evolved. In contrast, oleic acid content showed increasing trends until mid-maturity (sample Car C), followed by a slight decrease at the following maturity stages (samples Car D-F). Finally, linoleic acids exhibited fluctuating trends with the highest and lowest content being observed in samples B and E, respectively. SFAs and MUFAs were the most abundant class of fatty acids due to the high content of palmitic and linoleic acids, respectively. Moreover, the recorded values of PUFA/SFA ratio were higher than 0.45 in samples $B, D$ and $F$, whereas the values of $n 6 / n 3$ ratio were below 4.0 in samples A, B and E.

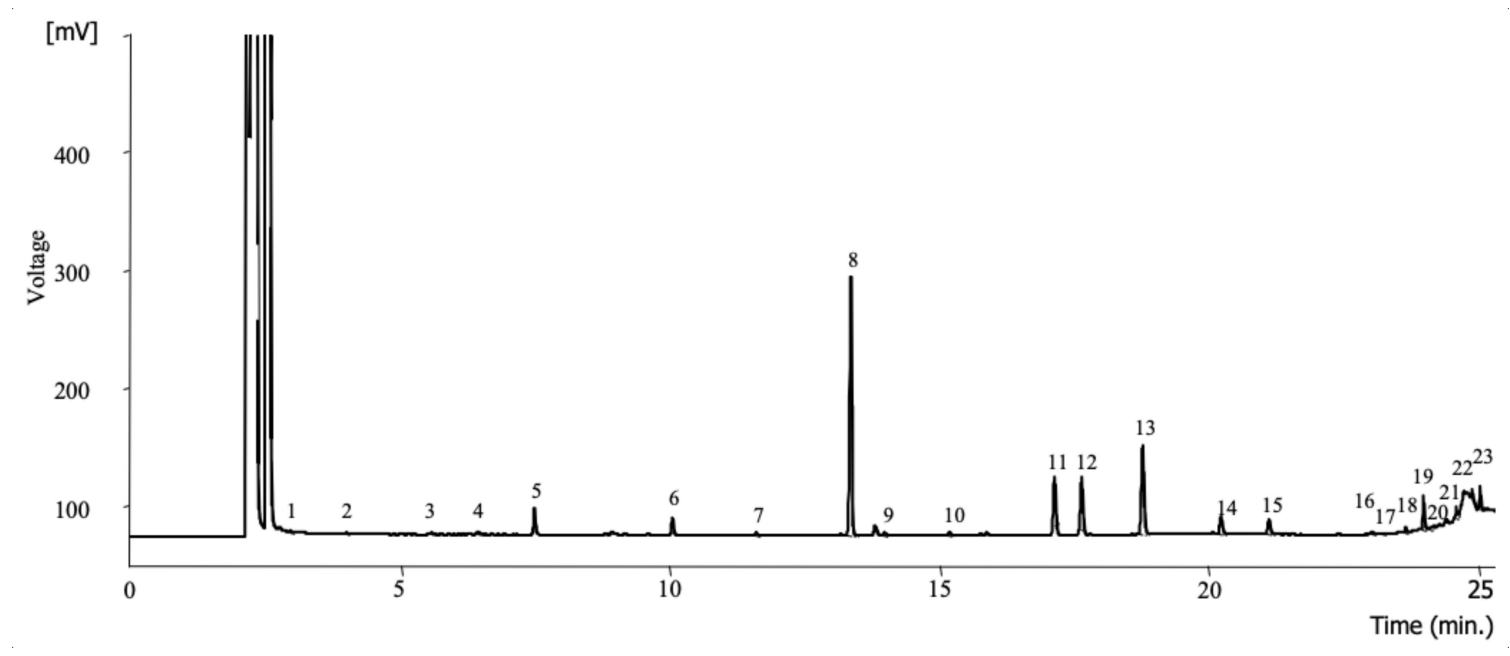

Figure 3. Chromatogram of fatty acids profile of Cynara cardunculus heads (sample Car A). 1. C6:0-caproic acid; 2. C8:0-caprylic acid; 3. C10:0-capric acid; 4. C11:0-undecanoic acid; 5. C12:0-lauric acid; 6. C14:0-myristic acid; 7. C15:0-pentadecanoic acid; 8. C16:0-palmitic acid; 9. C16:1-palmitoleic acid; 10. C17:0-heptadecanoic acid; 11. C18:0-stearic acid; 12. C18:1n9-oleic acid; 13. C18:2n6c-linoleic acid; 14. C18:3n3-alpha-linolenic acid; 15. C20:0-arachidic acid; 16. C21:0-heneicosanoic acid; 17. C20:3n6-eicosatrienoic acid; 18. C20:3n3-11,14,17-eicosatrienoic acid; 19. C22:1-erucic acid; 20. C20:5n3-eicosapentaenoic acid; 21. C22:2-docosadienoic acid; 22. C23:0-tricosanoic acid; 23. C24:0-lignoceric acid. 
Table 2. Fatty acids composition (relative \%) of Cynara cardunculus heads in relation to maturity stage $($ mean $\pm \mathrm{SD} ; \mathrm{n}=3)$.

\begin{tabular}{|c|c|c|c|c|c|c|}
\hline & Car A & Car B & Car C & Car D & Car E & Car F \\
\hline \multicolumn{7}{|c|}{ Fatty Acids (Relative Percentage, \%) } \\
\hline C6:0 & $0.49 \pm 0.01^{c}$ & $0.082 \pm 0.006^{\mathrm{f}}$ & $0.123 \pm 0.001^{\mathrm{d}}$ & $0.094 \pm 0.002 \mathrm{e}$ & $3.71 \pm 0.01^{\mathrm{a}}$ & $0.84 \pm 0.02^{b}$ \\
\hline C8:0 & $0.250 \pm 0.002^{c}$ & $0.19 \pm 0.01 \mathrm{~d}$ & $0.057 \pm 0.0033^{\mathrm{e}}$ & $0.059 \pm 0.006^{\mathrm{e}}$ & $1.314 \pm 0.004^{\mathrm{a}}$ & $0.29 \pm 0.01 \mathrm{~b}$ \\
\hline $\mathrm{C} 10: 0$ & $0.205 \pm 0.008^{\mathrm{cd}}$ & $0.198 \pm 0.001^{\mathrm{d}}$ & $0.254 \pm 0.006^{\mathrm{b}}$ & $0.21 \pm 0.02^{c}$ & $0.473 \pm 0.006^{\mathrm{a}}$ & $0.186 \pm 0.002^{e}$ \\
\hline $\mathrm{C} 11: 0$ & $0.72 \pm 0.02^{\mathrm{a}}$ & $0.335 \pm 0.001^{c}$ & $0.237 \pm 0.001^{\mathrm{d}}$ & $0.16 \pm 0.02^{\mathrm{e}}$ & $0.579 \pm 0.005^{b}$ & $0.17 \pm 0.01^{\mathrm{e}}$ \\
\hline $\mathrm{C} 12: 0$ & $1.8 \pm 0.1 \mathrm{~b}$ & $2.57 \pm 0.04^{\mathrm{a}}$ & $0.406 \pm 0.002^{\mathrm{e}}$ & $0.82 \pm 0.07^{\mathrm{d}}$ & $1.53 \pm 0.07^{\mathrm{c}}$ & $0.326 \pm 0.004^{\mathrm{f}}$ \\
\hline C13:0 & n.d. & $0.028 \pm 0.001 \mathrm{~d}$ & $0.0375 \pm 0.0007^{b}$ & $0.084 \pm 0.004^{a}$ & $0.030 \pm 0.001^{c}$ & $0.027 \pm 0.003^{d}$ \\
\hline C14:0 & $1.90 \pm 0.02^{b}$ & $0.58 \pm 0.02^{f}$ & $1.27 \pm 0.02^{\mathrm{d}}$ & $1.450 \pm 0.002^{c}$ & $2.69 \pm 0.01^{\mathrm{a}}$ & $1.0 \pm 0.1^{\mathrm{e}}$ \\
\hline C14:1 & n.d. & $0.53 \pm 0.01^{b}$ & n.d. & n.d. & $0.54 \pm 0.01$ a & $0.23 \pm 0.01^{c}$ \\
\hline $\mathrm{C} 15: 0$ & $0.48 \pm 0.01^{\mathrm{a}}$ & n.d. & $0.193 \pm 0.001^{\mathrm{d}}$ & $0.176 \pm 0.009^{\mathrm{e}}$ & $0.427 \pm 0.006^{b}$ & $0.28 \pm 0.02^{c}$ \\
\hline $\mathrm{C} 15: 1$ & n.d. & n.d. & n.d. & n.d. & $1.36 \pm 0.02^{*}$ & $0.122 \pm 0.003 *$ \\
\hline $\mathrm{C} 16: 0$ & $43.8 \pm 0.1^{\mathrm{a}}$ & $30.4 \pm 0.8^{\mathrm{b}}$ & $22.577 \pm 0.003^{\mathrm{d}}$ & $14.62 \pm 0.03^{\mathrm{f}}$ & $25.60 \pm 0.08^{c}$ & $17.8 \pm 0.2^{\mathrm{e}}$ \\
\hline $\mathrm{C} 16: 1$ & $0.43 \pm 0.01^{\mathrm{e}}$ & $0.317 \pm 0.003^{f}$ & $0.827 \pm 0.002^{d}$ & $12.62 \pm 0.03^{b}$ & $12.76 \pm 0.03^{a}$ & $6.69 \pm 0.04^{c}$ \\
\hline $\mathrm{C} 17: 0$ & $0.779 \pm 0.001^{\mathrm{a}}$ & $0.666 \pm 0.001^{b}$ & $0.313 \pm 0.003^{\mathrm{e}}$ & $0.239 \pm 0.001^{f}$ & $0.462 \pm 0.001^{\mathrm{d}}$ & $0.579 \pm 0.004^{c}$ \\
\hline C18:0 & $6.0 \pm 0.1^{\mathrm{a}}$ & $2.96 \pm 0.05^{\mathrm{e}}$ & $3.236 \pm 0.008^{d}$ & $2.687 \pm 0.004^{f}$ & $5.68 \pm 0.01^{b}$ & $4.599 \pm 0.001^{\mathrm{c}}$ \\
\hline $\mathrm{C} 18: \ln 9$ & $7.7 \pm 0.1^{\mathrm{e}}$ & $4.48 \pm 0.04^{f}$ & $46.6 \pm 0.1^{\mathrm{a}}$ & $32.8 \pm 0.1^{\mathrm{c}}$ & $32.47 \pm 0.08^{\mathrm{d}}$ & $33.7 \pm 0.8^{\mathrm{b}}$ \\
\hline $\mathrm{C} 18: 2 \mathrm{n} 6 \mathrm{c}$ & $20.1 \pm 0.1^{d}$ & $30.6 \pm 0.4^{\mathrm{a}}$ & $6.23 \pm 0.03^{\mathrm{e}}$ & $25.82 \pm 0.08^{c}$ & $0.748 \pm 0.002^{f}$ & $27.2 \pm 0.4^{b}$ \\
\hline C18:3n6 & n.d. & $0.176 \pm 0.006^{\mathrm{a}}$ & $0.049 \pm 0.004^{\mathrm{d}}$ & n.d. & $0.067 \pm 0.001^{c}$ & $0.145 \pm 0.005^{b}$ \\
\hline C18:3n3 & $5.55 \pm 0.05^{b}$ & $7.5 \pm 0.1^{\mathrm{a}}$ & $1.02 \pm 0.01^{\mathrm{e}}$ & $2.705 \pm 0.008^{c}$ & $0.3675 \pm 0.0007^{f}$ & $1.38 \pm 0.06^{\mathrm{d}}$ \\
\hline C20:0 & $2.18 \pm 0.02^{b}$ & $3.225 \pm 0.005^{\mathrm{a}}$ & $0.655 \pm 0.007^{\mathrm{e}}$ & $0.377 \pm 0.001^{f}$ & $0.882 \pm 0.009^{c}$ & $0.672 \pm 0.005^{\mathrm{d}}$ \\
\hline C20:1 & n.d. & $0.159 \pm 0.002^{c}$ & $0.196 \pm 0.004^{b}$ & $0.114 \pm 0.006^{\mathrm{e}}$ & $4.52 \pm 0.01 \mathrm{a}$ & $0.138 \pm 0.001 \mathrm{~d}$ \\
\hline $\mathrm{C} 20: 2$ & n.d. & $0.223 \pm 0.001^{b}$ & $0.107 \pm 0.001^{d}$ & $0.182 \pm 0.005^{c}$ & $0.0845 \pm 0.0007^{\mathrm{e}}$ & $0.31 \pm 0.02^{\mathrm{a}}$ \\
\hline $\mathrm{C} 21: 0$ & $0.276 \pm 0.002^{b}$ & $0.324 \pm 0.004^{a}$ & $0.092 \pm 0.004 \mathrm{e}^{\mathrm{e}}$ & $0.070 \pm 0.001^{\mathrm{f}}$ & $0.2695 \pm 0.0007^{c}$ & $0.169 \pm 0.005^{\mathrm{d}}$ \\
\hline C20:3n6 & $0.23 \pm 0.02^{\mathrm{b}}$ & $8.9 \pm 0.3^{a}$ & $0.103 \pm 0.009^{c}$ & $0.101 \pm 0.006^{c}$ & n.d. & n.d. \\
\hline $\mathrm{C} 20: 3 \mathrm{n} 3$ & $1.14 \pm 0.04^{b}$ & $0.142 \pm 0.001^{\mathrm{d}}$ & $0.12 \pm 0.01^{\mathrm{d}}$ & $1.38 \pm 0.08^{\mathrm{a}}$ & n.d. & $0.22 \pm 0.02^{c}$ \\
\hline $\mathrm{C} 22: 0$ & n.d. & $0.81 \pm 0.01 \mathrm{~d}$ & $2.6365 \pm 0.0007^{a}$ & $1.56 \pm 0.08^{c}$ & $1.645 \pm 0.004^{b}$ & n.d. \\
\hline $\mathrm{C} 22: 1$ & $2.249 \pm 0.008^{b}$ & $0.12 \pm 0.01^{f}$ & $4.9505 \pm 0.0007^{\mathrm{a}}$ & $1.22 \pm 0.02^{c}$ & $0.44 \pm 0.01 \mathrm{e}$ & $0.82 \pm 0.06^{\mathrm{d}}$ \\
\hline $\mathrm{C} 20: 5 \mathrm{n} 3$ & $0.38 \pm 0.04^{c}$ & n.d. & $0.036 \pm 0.001 \mathrm{e}$ & $0.32 \pm 0.03^{d}$ & $0.6285 \pm 0.002^{a}$ & $0.51 \pm 0.01^{b}$ \\
\hline $\mathrm{C} 22: 2$ & $0.30 \pm 0.01^{*}$ & $0.184 \pm 0.001 *$ & n.d. & n.d. & n.d. & n.d. \\
\hline C23:0 & $1.61 \pm 0.09^{\mathrm{a}}$ & $1.47 \pm 0.09 \mathrm{~b}$ & $0.26 \pm 0.02 \mathrm{e}$ & $0.2615 \pm 0.0007^{e}$ & $0.308 \pm 0.001 \mathrm{~d}$ & $0.52 \pm 0.02^{c}$ \\
\hline $\mathrm{C} 24: 0$ & $1.4 \pm 0.1^{c}$ & $2.88 \pm 0.05^{b}$ & $7.411 \pm 0.001^{\mathrm{a}}$ & n.d. & $0.413 \pm 0.001 \mathrm{e}$ & $1.1 \pm 0.1 \mathrm{~d}$ \\
\hline SFA & $61.9 \pm 0.4^{\mathrm{a}}$ & $46.7 \pm 0.7^{b}$ & $39.75 \pm 0.06^{\mathrm{d}}$ & $22.9 \pm 0.2^{\mathrm{e}}$ & $46.0 \pm 0.1^{c}$ & $28.5 \pm 0.5^{f}$ \\
\hline MUFA & $10.4 \pm 0.1^{\mathrm{e}}$ & $5.61 \pm 0.04^{f}$ & $52.6 \pm 0.1^{\mathrm{a}}$ & $46.73 \pm 0.07^{c}$ & $52.1 \pm 0.1^{\mathrm{b}}$ & $41.7 \pm 0.9^{\mathrm{d}}$ \\
\hline PUFA & $27.7 \pm 0.3^{\mathrm{d}}$ & $47.7 \pm 0.8^{\mathrm{a}}$ & $7.66 \pm 0.05^{\mathrm{e}}$ & $30.4 \pm 0.1^{\mathrm{b}}$ & $1.895 \pm 0.006^{\mathrm{f}}$ & $29.8 \pm 0.4^{c}$ \\
\hline PUFA/SFA & $0.45 \pm 0.01 \mathrm{~d}$ & $1.02 \pm 0.03^{c}$ & $0.193 \pm 0.001 \mathrm{e}$ & $1.33 \pm 0.01 \mathrm{a}$ & $0.0412 \pm 0.0002^{f}$ & $1.043 \pm 0.002^{b}$ \\
\hline$n-6 / n-3$ & $2.76 \pm 0.04^{d}$ & $1.88 \pm 0.02^{\mathrm{e}}$ & $5.02 \pm 0.04^{\mathrm{c}}$ & $5.8 \pm 0.1^{b}$ & $0.902 \pm 0.002^{\mathrm{f}}$ & $13.1 \pm 0.3^{\mathrm{a}}$ \\
\hline
\end{tabular}

Results are presented as mean \pm standard deviation. Concentration values are given as Supplementary Materials (Table S1). Different letters in the same line correspond to significant differences according to Tukey's honest significance test (HSD) test $(p<0.05)$. Fatty acids are expressed as relative percentage of each fatty acid. dw-dry weight; n.d.-not detected; C6:0-caproic acid; C8:0-caprylic acid; C10:0-capric acid; C11:0-undecanoic acid; C12:0-lauric acid; C13:0-tridecanoic acid; C14:0-myristic acid; C14:1-tetradecanoic acid; C15:0-pentadecanoic acid; C15:1-pentadecenoic acid; C16:0-palmitic acid; C16:1-palmitoleic acid; C17:0-heptadecanoic acid; C18:0-stearic acid; C18:1n9-oleic acid; C18:2n6c-linoleic acid; C18:3n6-gamma-linolenic acid; C18:3n3-alpha-linolenic acid; C20:0-arachidic acid; C20:1-gondoic acid; C20:2-eicosadienoic acid; C21:0-heneicosanoic acid; C20:3n6-eicosatrienoic acid; C20:3n3-11,14,17-eicosatrienoic acid; C22:0-behenic acid; C22:1-erucic acid; C20:5n3-eicosapentaenoic acid; C22:2-docosadienoic acid; C23:0-tricosanoic acid; C24:0-lignoceric acid; SFA-saturated fatty acids; MUFA-monounsaturated fatty acids; PUFA-polyunsaturated fatty acids; n-6/n-3: ratio of omega 6/omega 3 fatty acids. * Means statistical differences obtained by the t-student test, $p$-value $<0.01$.

\subsection{Organic Acids and Free Sugars}

In Table 3 are presented the results regarding the content in organic acids of Cynara cardunculus heads, in relation to maturity stage. The main detected organic acids were oxalic, quinic and malic acid, followed by citric and fumaric acids which were detected in lower amounts. Moreover, a great variation in individual organic acids content was observed at different maturity stages. In particular, at early stages (samples Car A and B) malic acid was the most abundant organic acid (1.45 and $2.31 \mathrm{~g} / 100 \mathrm{~g} \mathrm{dw}$, respectively), while at late stages (sample F) oxalic acid was the richest organic acid (12.1 g/100 g dw) followed by quinic acid $(3.3 \mathrm{~g} / 100 \mathrm{~g} \mathrm{dw})$. 
Table 3. Organic acids composition ( $\mathrm{g} / 100 \mathrm{~g} \mathrm{dw}$ ) of Cynara cardunculus heads in relation to maturity stage (mean $\pm \mathrm{SD} ; \mathrm{n}=3$ ).

\begin{tabular}{|c|c|c|c|c|c|c|}
\hline \multicolumn{7}{|c|}{ Organic Acids (g/100 g dw) } \\
\hline & Car A & Car B & Car C & Car D & Car E & Car F \\
\hline Oxalic acid & $0.324 \pm 0.002^{f}$ & $0.98 \pm 0.01^{b}$ & $0.3994 \pm 0.0001^{\mathrm{e}}$ & $0.650 \pm 0.001^{c}$ & $12.1 \pm 0.1^{a}$ & $0.44 \pm 0.01 \mathrm{~d}$ \\
\hline Quinic acid & $0.87 \pm 0.04^{b}$ & $0.46 \pm 0.01^{c}$ & $0.17 \pm 0.01^{\mathrm{e}}$ & $0.017 \pm 0.001^{\mathrm{f}}$ & $3.3 \pm 0.1^{\mathrm{a}}$ & $0.45 \pm 0.02^{\mathrm{d}}$ \\
\hline Malic acid & $1.45 \pm 0.06^{c}$ & $2.31 \pm 0.02^{a}$ & $1.495 \pm 0.001^{b}$ & $0.0149 \pm 0.0005^{\mathrm{e}}$ & $0.36 \pm 0.02^{d}$ & n.d. \\
\hline Citric acid & $0.70 \pm 0.04^{\mathrm{b}}$ & $0.86 \pm 0.05^{\mathrm{a}}$ & $0.66 \pm 0.02^{c}$ & $0.84 \pm 0.01^{\mathrm{a}}$ & $\operatorname{tr}$ & n.d. \\
\hline Fumaric acid & $0.046 \pm 0.001^{b}$ & $0.0542 \pm 0.0003^{\mathrm{a}}$ & $0.0110 \pm 0.0003^{c}$ & $\operatorname{tr}$ & $0.0045 \pm 0.0001^{\mathrm{d}}$ & n.d. \\
\hline Total & $3.39 \pm 0.06^{c}$ & $4.67 \pm 0.06^{b}$ & $2.74 \pm 0.03^{\mathrm{d}}$ & $1.52 \pm 0.01^{\mathrm{e}}$ & $15.7 \pm 0.2^{\mathrm{a}}$ & $0.89 \pm 0.01^{f}$ \\
\hline
\end{tabular}

Results are presented as mean \pm standard deviation. Different letters in the same line correspond to significant differences according to Tukey's HSD test $(p<0.05)$. dw-dry weight; tr-traces (below limit of quantification (LOQ) values); n.d.-not detected (below limit of detection (LOD) values). Calibration curves for organic acids: oxalic acid $\left(\mathrm{y}=1 \times 10^{6} \mathrm{x}+231891, \mathrm{R}^{2}=0.9999 ; \mathrm{LOD}=12.55 \mu \mathrm{g} / \mathrm{mL} ; \mathrm{LOQ}=41.82 \mu \mathrm{g} / \mathrm{mL}\right) ;$ quinic acid $(\mathrm{y}=671557 \mathrm{x}$ $\left.+14583, \mathrm{R}^{2}=0.9998 ; \mathrm{LOD}=24.18 \mu \mathrm{g} / \mathrm{mL} ; \mathrm{LOQ}=80.61 \mu \mathrm{g} / \mathrm{mL}\right) ;$ malic acid $\left(\mathrm{y}=950041 \mathrm{x}+6255.6, \mathrm{R}^{2}=0.9999 ;\right.$ $\mathrm{LOD}=35.76 \mu \mathrm{g} / \mathrm{mL} ; \mathrm{LOQ}=119.18 \mu \mathrm{g} / \mathrm{mL}) ;$ citric acid $\left(\mathrm{y}=1 \times 10^{5} \mathrm{x}+10277, \mathrm{R}^{2}=0.9997 ; \mathrm{LOD}=10.47 \mu \mathrm{g} / \mathrm{mL}\right.$; $\mathrm{LOQ}=34.91 \mu \mathrm{g} / \mathrm{mL})$ and fumaric acid $\left(\mathrm{y}=1 \times 10^{7} \mathrm{x}+614399, \mathrm{R}^{2}=0,9986 ; \mathrm{LOD}=0.08 \mu \mathrm{g} / \mathrm{mL} ; \mathrm{LOQ}=0.26 \mu \mathrm{g} / \mathrm{mL}\right)$.

The free sugars composition of cardoon heads in relation to maturity stage is presented in Table 4. Sucrose, glucose and raffinose were the main detected sugars, followed by fructose and trehalose, while a great variation in sugar composition was observed in response to maturity stage. In particular, sample Car $C$ had the highest content in total sugars $(7.4 \mathrm{~g} / 100 \mathrm{~g} \mathrm{dw})$, with raffinose and fructose (1.8 and $1.64 \mathrm{mg} / 100 \mathrm{~g} \mathrm{dw}$, respectively) being present in higher amounts; the same sample was also the only one containing all the detected sugars. Samples Car D and Car E revealed the lowest content of total sugars $(1.03 \mathrm{mg} / 100 \mathrm{~g} \mathrm{dw})$. Moreover, in the most advanced maturation stages (samples Car D-F) trehalose was the free sugar being recorded in higher amounts $(0.34-0.96 \mathrm{mg} / 100 \mathrm{~g} \mathrm{dw})$, whereas raffinose was not detected. In the remaining samples car $B$ and $C$, the presence of raffinose and sucrose stands out, except for sample Car B where sucrose was not detected.

Table 4. Free sugars composition $(\mathrm{g} / 100 \mathrm{~g} \mathrm{dw})$ of Cynara cardunculus heads in relation to maturity stage (mean $\pm \mathrm{SD} ; \mathrm{n}=3$ ).

\begin{tabular}{|c|c|c|c|c|c|c|}
\hline \multicolumn{7}{|c|}{ Free Sugars $(\mathrm{g} / 100 \mathrm{~g}$ dw) } \\
\hline Fructose & $0.13 \pm 0.03^{\mathrm{d}}$ & $0.51 \pm 0.04^{b}$ & $1.64 \pm 0.06^{\mathrm{a}}$ & $0.013 \pm 0.004^{\mathrm{e}}$ & $0.14 \pm 0.02^{d}$ & $0.184 \pm 0.001^{c}$ \\
\hline Sucrose & $2.39 \pm 0.06^{b}$ & n.d. & $3.0 \pm 0.1^{\mathrm{a}}$ & n.d. & $0.28 \pm 0.02^{c}$ & $0.11 \pm 0.01^{\mathrm{d}}$ \\
\hline Trehalose & $0.23 \pm 0.04^{\mathrm{d}}$ & $0.98 \pm 0.02^{\mathrm{a}}$ & $0.26 \pm 0.02^{d}$ & $0.96 \pm 0.09^{a}$ & $0.34 \pm 0.02^{b}$ & $0.797 \pm 0.005^{c}$ \\
\hline Raffinose & $2.24 \pm 0.07^{b}$ & $2.62 \pm 0.06^{\mathrm{a}}$ & $1.8 \pm 0.1^{\mathrm{c}}$ & n.d. & n.d. & n.d. \\
\hline
\end{tabular}

Results are presented as mean \pm standard deviation. Different letters correspond to significant differences according to Tukey's. HSD test $(p<0.05)$. dw-dry weight; n.d.-not detected (bellow limit of detection (LOD) values). Free sugars calibration curves: fructose $\left(y=1.04 x, R^{2}=0.999 ; \mathrm{LOD}=0.05 \mathrm{mg} / \mathrm{mL}\right)$, glucose $(\mathrm{y}=0.935 \mathrm{x}$, $\mathrm{R}^{2}=0.999 ; \mathrm{LOD}=0.08 \mathrm{mg} / \mathrm{mL}$; limit of quantification $\left.(\mathrm{LOQ})=0.25 \mathrm{mg} / \mathrm{mL}\right)$ and trehalose $\left(\mathrm{y}=0.991 \mathrm{x}, \mathrm{R}^{2}=0.999\right.$; $\mathrm{LOD}=0.07 \mathrm{mg} / \mathrm{mL}, \mathrm{LOQ}=0.24 \mathrm{mg} / \mathrm{mL}$ ).

\subsection{Principal Component Analysis (PCA)}

Principal component analysis (PCA) is used to reduce multivariate data complexity as a method of identifying patterns and expressing data in ways that highlight similarities and differences, and further identify groups of samples according to their maturation stage [31,32]. The first four principal components (PCs) were associated with Eigen values higher than 1 and explained $97.2 \%$ of the cumulative variance, with PC1 accounting for $43.8 \%$, PC2 for $22.9 \%$, PC3 for $19.4 \%$ and finally PC4 for $11.1 \%$. PC1 was positively correlated to lipidic fraction, raffinose and total sugars, malic, citric and fumaric acid, linoleic and $\alpha$-linolenic acid and PUFA content, whereas it was negatively correlated to oxalic and quinic acid, total organic acids and MUFA content. PC2 was positively correlated to sucrose, quinic acid, palmitic and oleic acid and SFA content, whereas it was negatively correlated to trehalose, 
linoleic and $\alpha$-linolenic acid and PUFA. Similarly, PC3 was positively correlated to $\alpha$-tocopherol, tregalose and oxalic acid content, whereas it was negatively correlated to fructose, sucrose, raffinose, total sugars, malic and citric acid and oleic acid. Finally, PC4 was positively correlated to fructose, sucrose, total organic acids, oleic acid and MUFA content, whereas it was negatively correlated to palmitic and stearic acid and SFA content. These results indicating a correct application of the PCA allowing differentiation between the tested maturity stages, as shown in the corresponding scatterplot (Figure 4). Moreover, the plot suggests that the differences in the chemical composition of the tested samples are correlated with the maturation stage. The early (sampes Car A and B) and mid and late stages (samples Car D and F) are closely positioned, whereas samples Car C and Car E are clearly distinct due to the very low $\alpha$-tocopherol and very high organic acids content, respectively, compared to the rest of the tested maturity stages. The loading plot (Figure 5) of the first two components revealed groups of positively correlated variables, namely the upper right quadrant comprising fructose, sucrose, raffinose, total sugars, lipidic fraction, SFA, palmitic acid, malic acid and fumaric acid; the lower right quadrant comprising tocopherols, glucose, citric acid, PUFA and and linoleic acid; the upper left quadrant comprising quinic acid, oxalic acid, total organic acids and oleic acid; the lower left quadant comprising trehalose and $\alpha$-linolenic acid

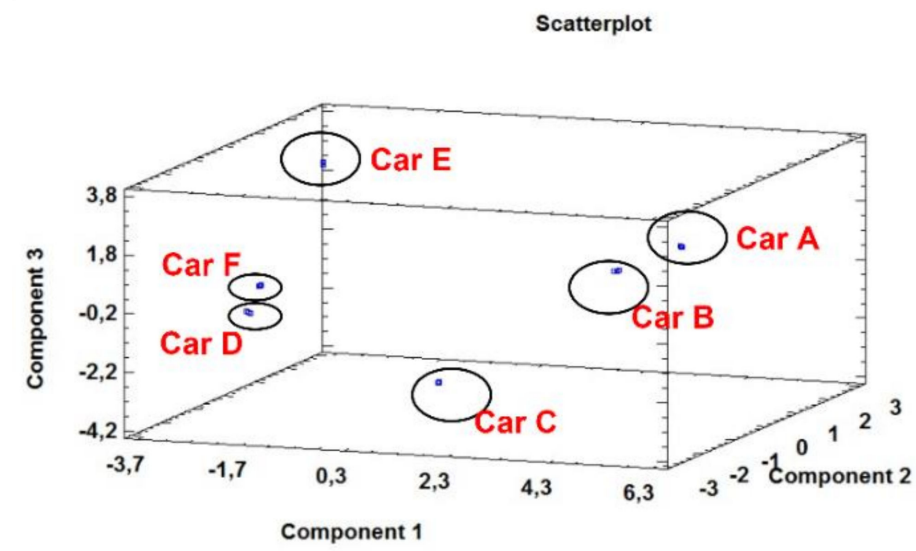

Figure 4. Three dimensional principal components scatterplot of the tested variables at different maturation stages of cardoon heads (samples Car A-F).

Plot of Component Weights

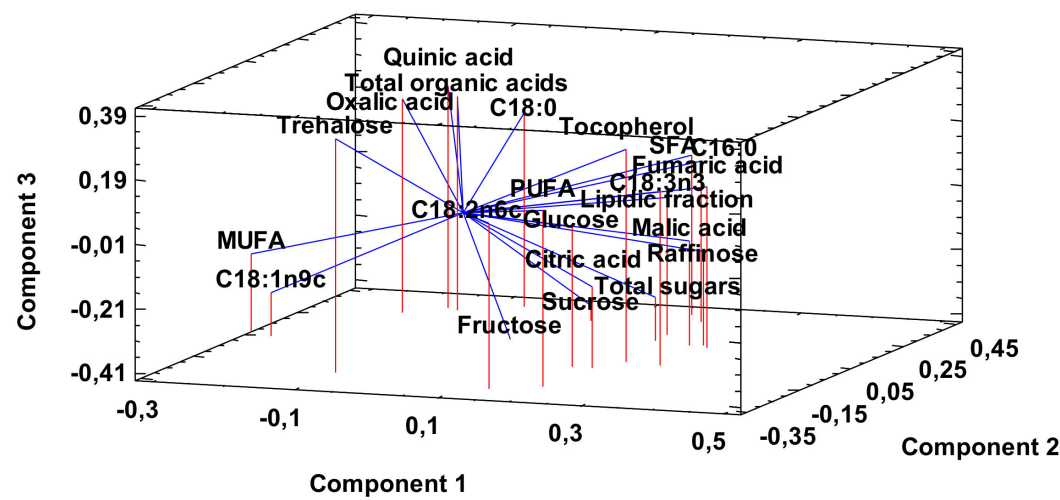

Figure 5. The principal components loading plot of the tested variables at different maturation stages of cardoon heads. SFA-saturated fatty acids, MUFA-Monounsaturated fatty acids, PUFA-polyunsaturated fatty acids. 


\section{Discussion}

It could be suggested that the maturation stage of cardoon heads affects the fatty acids content which do not present a similar qualitative and quantitative profile over the maturation process. For example, pentadecanoic acid (C15:1) was detected only in senescent head samples (Car E and F), whereas docosadienoic acid (C22:2) was only detected in immature heads (samples Car A and B). The fatty acid profile of cardoon has already been studied by our team who studied cardoon seeds collected at full maturity and when heads were dry and senesced and the influence of harvesting time was suggested [33]. Moreover, harvesting time is also associated with variable climate conditions as shown in Figure 2, which according to the literature may also affect the compositional profile of globe artichoke [20]. Therefore, the dry conditions during the period of March-May in our study could be implicated in the observed differences among the tested maturation stages. Analogous fatty acids composition in cardoon heads has also been previously described by Petropoulos et al. [34], who studied different cardoon genotypes and verified that palmitic and linoleic acids were present in higher abundance in all the studied heads. Palmitic and linoleic acids were also suggested as the main fatty acid in globe artichoke heads by Dosi et al. [35] although they reported significantly higher amounts of linoleic than palmitic acid ( 55.20 and $34.80 \mathrm{mg} / 100 \mathrm{~g} \mathrm{fw}$, respectively) compared to our study. The studies of the lipidic fraction in cardoon tissues mostly refer to its seeds [36-38], as a result of the great interest for its industrial potential for the biodiesel production, although fatty acids composition of stalks, capitula and leaves has been also reported [39]. The high content in fatty acids present in cardoon heads, particularly the essential linoleic and oleic acids, is an added value to this multi-purpose crop that could be used for the production of these acids in industrial scale [40]. To the best of our knowledge, this is the first report that evaluated the influence of the physiological stages of cardoon heads collected during all the flowering stage.

Regarding fatty acids classification, our results showed that the saturated fatty acids (SFA) were the most abundant class of fatty acids in heads of early maturity (Car A-C), whereas monounsaturated fatty acids (MUFA) were the predominant ones in the remaining samples (samples D-F). Furthermore, the maturation stage of the samples reveals a strong influence both on the fatty acids profile, as well as on the proportion of PUFA/SFA and n-6/n-3, parameters which are associated with the nutritional value and the functional properties of food products $[33,41]$. Sample Car B was the only sample with a PUFA/SFA value higher than 0.45 and an $n-6 / n-3$ ratio value lower than 4.0 , both characteristics being associated with good nutritional properties. This evidence agrees with the popular medicine, since in the Mediterranean cousin cardoon heads are used when immature [19,42,43]. With the results obtained in this study, we corroborate the consumption of immature cardoon heads, preferably harvested in the beginning of May, based on the conditions of the growing location.

The tocopherols content detected in cardoon heads is presented in Table 1. The $\alpha$-tocopherol was the only isoform identified and was detected in all the studied samples. Tocopherols are antioxidant compounds with high capacity to undergo oxidation reactions, therefore fluctuations of environmental conditions that may induce plant stress throughout the growth cycle of the heads could be a justification for the observed fluctuations in tocopherols content [23]. As shown in Figure 2, variable climatic conditions prevailed during and prior the harvesting period in our study, especially a dry period during March-May which could be associated with stressful conditions that resulted in an increase of tocopherols content. In a previous study, we studied the bioactive properties of cardoon heads also analyzed in the present work and despite the antioxidant capacity associated with tocopherols, the anticipation that the sample Car B with the highest tocopherols content would show the greater antioxidant activity was not verified. This fact suggests that other classes of compounds, such as phenolic compounds, could be also related to the demonstrated antioxidant potential [19], while Kukić et al. [44] suggested that $\beta$-sitosterol possessed a strong antioxidant capacity in extracts obtained from cardoon bracts. Despite that, the highest content of $\alpha$-tocopherol in this sample could be associated with the highest content in PUFAs (see Table 2) highlighting the protective effects of tocopherols against lipid peroxidation $[45,46]$. Similarly to tocopherols composition of heads, other 
plant tissues of cardoon do not present a wide variety of tocopherols isoforms and the $\alpha$-tocopherol was the only isoform detected in cardoon seeds [23,47], contrary to the cardoon seed oil which was rich in $\alpha$ - and $\delta$-tocopherols [47-50] and leaf blades that contained all tocopherol isoforms [51].

The organic acids profile (Table 3) was also different between samples with a great variation during the maturation process of the detected organic acids: oxalic, quinic, malic, citric, and fumaric. The sample collected at the principal growth stage (PGS) 7 (Car E) revealed the highest content in organic acids ( $15.7 \mathrm{mg} / 100 \mathrm{~g} \mathrm{dw})$, and sample Car F (PGS 8) the lowest abundance $(0.89 \mathrm{mg} / 100 \mathrm{~g} \mathrm{dw})$. Immature to mid maturity heads (samples Car A-C) had malic acid as the most abundant organic acid $(1.45-2.31 \mathrm{mg} / 100 \mathrm{~g} \mathrm{dw})$. In sample $\mathrm{D}$, the organic acid present in higher quantities was citric acid $(0.84 \mathrm{~g} / 100 \mathrm{~g} \mathrm{dw})$, while in advanced maturation stages, oxalic and quinic acids were the organic acids present in higher amounts. Moreover, considering the concomitant decrease in total sugars content at late maturity stages, the increase of organic acids especially in sample Car E could be explained by the increased requirements in osmolytes as a mechanism of cardoon plants to overcome stress conditions induced by the high mean and max temperatures and the concomitant low precipitation (see Figure 2) [52,53]. Compared to other reports, cardoon heads revealed greater variety and lower abundance of organic acids than seeds [23,47], while organic acids profile was similar to aerial parts [54]. Finally, the high quinic acid in sample Car E could be valorized for its confirmed high antioxidant potential $[55,56]$.

The qualitative and quantitative information regarding the free sugars composition of cardoon heads are presented in Table 4. The samples analyzed revealed significant differences in free sugars content throughout the maturation process. Therefore, in early stages (sample car A) sucrose and raffinose were the main detected sugars, while sugar composition was altered in the following stage (sample car B) and raffinose and glucose were the most abundant sugars. Similarly, in sample $C$ sucrose, fructose and raffinose were detected in the highest amounts, whereas in the following stages (samples D-F) total sugars content was significantly reduced consisting mainly of trehalose. Considering that the tested samples were collected under environmental conditions [19] could justify the observed differences in sugar composition between the various maturity stages. According to Petropoulos et al. [34] who studied the influence of the genotype on different parameters of cardoon heads, significant differences between the genotypes tested were also suggested. Previous studies identified sucrose [31,34,57] and glucose [58,59] as the most abundant sugars. These differences with the present work could be justified by the effect of factors such as the geographic location and growing conditions [58] or the stage of maturity of the analyzed samples [60], especially at early maturity stages where heads are edible and sweet in taste. In the same context and considering that artichoke is a rich source of inulin [61,62], free sugars content at late maturity stages (samples car D-E) exhibits a significant reduction probably due to the inulin formation and the accumulation of storage carbohydrates $[63,64]$. This argument could also explain the high content of trehalose at late maturity stages where the amounts of other free sugars is reduced, while the evolving environmental conditions (increasing temperatures, water shortage) could pose plants under stress and increase the needs of osmolytes for stress tolerance [52,65]. Finally, the decrease of total free sugars at late maturity stages should be linked with the lignification process and the hard texture of cardoon heads, since lignin biosynthesis involves the binding of non-structural carbohydrates for the formation of lignin carbohydrate complexes (LCC) [65].

\section{Conclusions}

Cardoon heads are widely consumed in several dishes of the Mediterranean countries, such as salad and soups, due to their richness in health-promoting compounds and to their well-recognized nutritional value. However, the stage when heads are edible is relatively short and is highly affected by environmental conditions during harvesting that may result in heads of hard texture due to high fibers content which make them inedible. Therefore, a considerable number of heads are considered as waste with potential suggested uses the energy and biomass production. With the present study we aimed to 
analyze cardoon heads chemical composition and evaluate the potential of alternative uses that will increase the added value of the crop. Our results allowed a complete characterization of the chemical composition of cardoon heads and the evaluation of the effect that the maturity stage has on the lipid, fatty acids, tocopherols, organic acids, and free sugars content. To the best of our knowledge, this is the first report that studied the influence of the maturity stage on the composition in lipidic content, fatty acids, tocopherols, organic acids, and free sugars content. In particular, immature heads (samples Car A and B) exhibited the highest content in lipidic fraction and $\alpha$-tocopherol, respectively. Moreover, samples of mid- (sample Car C) and late maturity (sample Car E) had the highest total sugars and total organic acids content, respectively. With the present results we verified that the maturation stage of cardoon heads had a significant influence on the chemical composition. The obtained information should be implemented by different application areas, e.g., nutraceuticals and food supplements, in order to obtain the maximum potential of cardoon heads utilization, as well as to improve the production and commercialization techniques.

Supplementary Materials: The following are available online at http://www.mdpi.com/2073-4395/10/8/1088/s1, Table S1: Composition of fatty acids (mg/100 g dw) of Cynara cardunculus heads in relation to maturity 16 stage $($ mean $\pm \mathrm{SD} ; \mathrm{n}=3)$.

Author Contributions: Methodology, Investigation, Writing-Original Draft, F.M. and Â.F.; Methodology, Writing-Reviewing and Editing, S.A.P.; Conceptualization, Methodology, Writing-Reviewing and Editing, C.S.-B., I.C.F.R.F. and L.B. All authors have read and agreed to the published version of the manuscript.

Funding: This work was financially support by national funds FCT/MCTES to CIMO (UIDB/00690/2020). We also thank the FCT for the PhD grant (SFRH/BD/146614/2019) of F. Mandim. The authors are also grateful to the financial support though the TRANSCOLAB (0612_TRANS_CO_LAB_2_P).

Acknowledgments: To the Foundation for Science and Technology (FCT, Portugal) for the contracts of Â. Fernandes and L. Barros through the institutional scientific employment program.

Conflicts of Interest: The authors declare no conflict of interest.

\section{References}

1. Gominho, J.; Curt, M.D.; Lourenço, A.; Fernández, J.; Pereira, H. Cynara cardunculus L. as a biomass and multi-purpose crop: A review of 30 years of research. Biomass Bioenergy 2018, 109, 257-275. [CrossRef]

2. Zayed, A.; Serag, A.; Farag, M.A. Cynara cardunculus L.: Outgoing and potential trends of phytochemical, industrial, nutritive and medicinal merits. J. Funct. Foods 2020, 69, 103937. [CrossRef]

3. De Falco, B.; Incerti, G.; Amato, M.; Lanzotti, V. Artichoke: Botanical, agronomical, phytochemical, and pharmacological overview. Phytochem. Rev. 2015, 14, 993-1018. [CrossRef]

4. Gostin, A.I.; Waisundara, V.Y. Edible flowers as functional food: A review on artichoke (Cynara cardunculus L.). Trends Food Sci. Technol. 2019, 86, 381-391. [CrossRef]

5. Brás, T.; Paulino, A.F.C.; Neves, L.A.; Crespo, J.G.; Duarte, M.F. Ultrasound assisted extraction of cynaropicrin from Cynara cardunculus leaves: Optimization using the response surface methodology and the effect of pulse mode. Ind. Crops Prod. 2020, 150, 112395. [CrossRef]

6. Amira, A.B.; Besbes, S.; Attia, H.; Blecker, C. Milk-clotting properties of plant rennets and their enzymatic rheological, and sensory role in cheese making: A review. Int. J. Food Prop. 2017, 28, 576-593.

7. Gomes, S.; Belo, A.T.; Alvarenga, N.; Dias, J.; Lage, P.; Pinheiro, C.; Pinto-Cruz, C.; Brás, T.; Duarte, M.F.; Martins, A.P.L. Characterization of Cynara cardunculus L. flower from Alentejo as a coagulant agent for cheesemaking. Int. Dairy J. 2019, 91, 178-184. [CrossRef]

8. Jiménez-Moreno, N.; Cimminelli, M.J.; Volpe, F.; Ansó, R.; Esparza, I.; Mármol, I.; Rodríguez-Yoldi, M.J.; Ancín-Azpilicueta, C. Phenolic composition of Artichoke waste and its antioxidant capacity on differentiated Caco-2 cells. Nutrients 2019, 11, 1723. [CrossRef]

9. Pari, L.; Alfano, V.; Mattei, P.; Santangelo, E. Pappi of cardoon (Cynara cardunculus L.): The use of wetting during the harvesting aimed at recovering for the biorefinery. Ind. Crops Prod. 2017, 108, 722-728. [CrossRef]

10. Francaviglia, R.; Bruno, A.; Falcucci, M.; Farina, R.; Renzi, G.; Russo, D.E.; Sepe, L.; Neri, U. Yields and quality of Cynara cardunculus L. wild and cultivated cardoon genotypes. A case study from a marginal land in Central Italy. Eur. J. Agron. 2016, 72, 10-19. [CrossRef] 
11. Lourenço, A.; Neiva, D.M.; Gominho, J.; Curt, M.D.; Fernández, J.; Marques, A.V.; Pereira, H. Biomass production of four Cynara cardunculus clones and lignin composition analysis. Biomass Bioenergy 2015, 76, 86-95. [CrossRef]

12. Conceição, C.; Martins, P.; Alvarenga, N.; Dias, J.; Lamy, E.; Garrido, L.; Gomes, S.; Freiras, S.; Belo, A.; Brás, T.; et al. Cynara cardunculus: Use in cheesemaking and pharmaceutical applications. In Technological Approaches for Novel Applications in Dairy Processing; Intech Open: London, UK, 2016; Volume 1, pp. $73-107$.

13. D'Antuono, I.; Carola, A.; Sena, L.M.; Linsalata, V.; Cardinali, A.; Logrieco, A.F.; Colucci, M.G.; Apone, F. Artichoke polyphenols produce skin anti-age effects by improving endothelial cell integrity and functionality. Molecules 2018, 23, 2729. [CrossRef] [PubMed]

14. Salem, M.B.; Affes, H.; Athmouni, K.; Ksouda, K.; Dhouibi, R.; Sahnoun, Z.; Hammami, S.; Zeghal, K.M. Chemicals compositions, antioxidant and anti-inflammatory activity of Cynara scolymus leaves extracts, and analysis of major bioactive polyphenols by HPLC. Evid. Based Complement. Altern. Med. 2017, 2017, 4951937.

15. Marques, P.; Marto, J.; Gonçalves, L.M.; Pacheco, R.; Fitas, M.; Pinto, P.; Serralheiro, M.L.M.; Ribeiro, H. Cynara scolymus L.: A promising Mediterranean extract for topical anti-aging prevention. Ind. Crops Prod. 2017, 109, 699-706. [CrossRef]

16. Almeida, C.M.; Simões, I. Cardoon-based rennets for cheese production. Appl. Microbiol. Biotechnol. 2018, 102, 4675-4686. [CrossRef] [PubMed]

17. Faria-Silva, C.; Ascenso, A.; Costa, A.M.; Marto, J.; Carvalheiro, M.; Ribeiro, H.M.; Simões, S. Feeding the skin: A new trend in food and cosmetics convergence. Trends Food Sci. Technol. 2020, 95, 21-32. [CrossRef]

18. Dias, M.I.; Barros, L.; Barreira, J.C.M.; Alves, M.J.; Barracosa, P.; Ferreira, I.C.F.R. Phenolic profile and bioactivity of cardoon (Cynara cardunculus L.) inflorescence parts: Selecting the best genotype for food applications. Food Chem. 2018, 268, 196-202. [CrossRef]

19. Mandim, F.; Petropoulos, S.A.; Giannoulis, K.D.; Dias, M.I.; Fernandes, Â.; Pinela, J.; Kostic, M.; Soković, M.; Barros, L.; Santos-Buelga, C.; et al. Seasonal variation of bioactive properties and phenolic composition of Cynara cardunculus var. altilis. Food Res. Int. 2020, 134, 109281. [CrossRef]

20. Pandino, G.; Lombardo, S.; Lo Monaco, A.; Mauromicale, G. Choice of time of harvest influences the polyphenol profile of globe artichoke. J. Funct. Foods 2013, 5, 1822-1828. [CrossRef]

21. Scavo, A.; Pandino, G.; Restuccia, C.; Parafati, L.; Cirvilleri, G.; Mauromicale, G. Antimicrobial activity of cultivated cardoon (Cynara cardunculus L. var. altilis DC.) leaf extracts against bacterial species of agricultural and food interest. Ind. Crops Prod. 2019, 129, 206-211. [CrossRef]

22. Li, Y.; Kong, D.; Fu, Y.; Sussman, M.R.; Wu, H. The effect of developmental and environmental factors on secondary metabolites in medicinal plants. Plant. Physiol. Biochem. 2020, 148, 80-89. [CrossRef]

23. Mandim, F.; Dias, M.I.; Pinela, J.; Barracosa, P.; Ivanov, M.; Ferreira, I.C.F.R. Chemical composition and in vitro biological activities of cardoon (Cynara cardunculus L. var. altilis DC.) seeds as influenced by viability. Food Chem. 2020, 323, 126838. [CrossRef] [PubMed]

24. Ben Amira, A.; Blecker, C.; Richel, A.; Arias, A.A.; Fickers, P.; Francis, F.; Besbes, S.; Attia, H. Influence of the ripening stage and the lyophilization of wild cardoon flowers on their chemical composition, enzymatic activities of extracts and technological properties of cheese curds. Food Chem. 2018, 245, 919-925. [CrossRef] [PubMed]

25. Claus, T.; Maruyama, S.A.; Palombini, S.V.; Montanher, P.F.; Bonafé, E.G.; de Oliveira Santos Junior, O.; Matsushita, M.; Visentainer, J.V. Chemical characterization and use of artichoke parts for protection from oxidative stress in canola oil. LWT Food Sci. Technol. 2015, 61, 346-351. [CrossRef]

26. Archontoulis, S.V.; Struik, P.C.; Vos, J.; Danalatos, N.G. Phenological growth stages of Cynara cardunculus: Codification and description according to the BBCH scale. Ann. Appl. B 2013, 156, 253-270. [CrossRef]

27. Petropoulos, S.A.; Pereira, C.; Tzortzakis, N.; Barros, L.; Ferreira, I.C.F.R. Nutritional value and bioactive compounds characterization of plant parts from Cynara cardunculus L. (Asteraceae) cultivated in central Greece. Front. Plant. Sci. 2018, 9, 1-12. [CrossRef]

28. Barros, L.; Pereira, E.; Calhelha, R.C.; Dueñas, M.; Carvalho, A.M.; Santos-Buelga, C.; Ferreira, I.C.F.R. Bioactivity and chemical characterization in hydrophilic and lipophilic compounds of Chenopodium ambrosioides L. J. Funct. Foods 2013, 5, 1732-1740. [CrossRef]

29. Mandim, F.; Barros, L.; Calhelha, R.C.; Abreu, R.M.V.; Pinela, J.; Alves, M.J.; Heleno, S.; Santos, P.F.; Ferreira, I.C.F.R. Calluna vulgaris (L.) Hull: Chemical characterization, evaluation of its bioactive properties and effect on the vaginal microbiota. Food Funct. 2019, 10, 78-89. [CrossRef] 
30. Dias, M.I.; Barros, L.; Morales, P.; Sánchez-Mata, M.C.; Oliveira, M.B.P.P.; Ferreira, I.C.F.R. Nutritional parameters of infusions and decoctions obtained from Fragaria vesca L. roots and vegetative parts. LWT Food Sci. Technol. 2015, 62, 32-38. [CrossRef]

31. Pereira, E.; Barros, L.; Calhelha, R.C.; Dueñas, M.; Carvalho, A.M.; Santos-Buelga, C.; Ferreira, I.C.F.R. Bioactivity and phytochemical characterization of Arenaria Montana L. Food Funct. 2014, 5, 1848-1855. [CrossRef]

32. El-Nakhel, C.; Petropoulos, S.A.; Pannico, A.; Kyriacou, M.C.; Giordano, M.; Colla, G.; Troise, A.D.; Vitaglione, P.; De Pascale, S.; Rouphael, Y. The bioactive profile of lettuce produced in a closed soilless system as configured by combinatorial effects of genotype and macrocation supply composition. Food Chem. 2020, 309, 125713. [CrossRef] [PubMed]

33. Petropoulos, S.A.; Fernandes, Â.; Calhelha, R.C.; Danalatos, N.; Barros, L.; Ferreira, I.C.F.R. How extraction method affects yield, fatty acids composition and bioactive properties of cardoon seed oil? Ind. Crops Prod. 2018, 124, 459-465. [CrossRef]

34. Petropoulos, S.A.; Pereira, C.; Ntatsi, G.; Danalatos, N.; Barros, L.; Ferreira, I.C.F.R. Nutritional value and chemical composition of Greek artichoke genotypes. Food Chem. 2018, 267, 296-302. [CrossRef] [PubMed]

35. Dosi, R.; Daniele, A.; Guida, V.; Ferrara, L.; Severino, V.; Di Maro, A. Nutritional and metabolic profiling of the globe artichoke (Cynara scolymus L. "Capuanella" heads) in province of caserta, Italy. Aust. J. Crop. Sci. 2013, 7, 1927-1934.

36. Petropoulos, S.; Fernandes, Â.; Pereira, C.; Tzortzakis, N.; Vaz, J.; Soković, M.; Barros, L.; Ferreira, I.C.F.R. Bioactivities, chemical composition and nutritional value of Cynara cardunculus L. seeds. Food Chem. 2019, 289, 404-412. [CrossRef] [PubMed]

37. Alexandre, A.M.R.C.; Dias, A.M.A.; Seabra, I.J.; Portugal, A.A.T.G.; De Sousa, H.C.; Braga, M.E.M. Biodiesel obtained from supercritical carbon dioxide oil of Cynara cardunculus L. J. Supercrit. Fluids 2012, 68, 52-63. [CrossRef]

38. Raccuia, S.A.; Piscioneri, I.; Sharma, N.; Melilli, M.G. Genetic variability in Cynara cardunculus L. domestic and wild types for grain oil production and fatty acids composition. Biomass Bioenergy 2011, 35, 3167-3173. [CrossRef]

39. Ramos, P.A.B.; Guerra, A.R.; Guerreiro, O.; Freire, C.S.R.; Silva, A.M.S.; Duarte, M.F.; Silvestre, A.J.D. Lipophilic extracts of Cynara cardunculus L. var. altilis (DC): A source of valuable bioactive terpenic compounds. J. Agric. Food Chem. 2013, 61, 8420-8429. [CrossRef]

40. Petropoulos, S.A.; Perreira, C.; Barros, L.; Ferreira, I.C.F.R. Leaf parts from Greek artichoke genotypes as a good source of bioactive compounds and antioxidants. Food Funct. 2017, 8, 2022-2029. [CrossRef]

41. Liu, L.; Hu, Q.; Wu, H.; Xue, Y.; Cai, L.; Fang, M.; Liu, Z.; Yao, P.; Wu, Y.; Gong, Z. Protective role of n6/n3 PUFA supplementation with varying DHA/EPA ratios against atherosclerosis in mice. J. Nutr. Biochem. 2016, 32, 171-180. [CrossRef]

42. Ierna, A.; Mauromicale, G. Cynara cardunculus L. genotypes as a crop for energy purposes in a Mediterranean environment. Biomass Bioenergy 2010, 34, 754-760. [CrossRef]

43. Pandino, G.; Lombardo, S.; Mauromicale, G.; Williamson, G. Profile of polyphenols and phenolic acids in bracts and receptacles of globe artichoke (Cynara cardunculus var. scolymus) germplasm. J. Food Compos. Anal. 2011, 24, 148-153. [CrossRef]

44. Kukić, J.; Popovic, V.; Petrović, S.; Mucaji, P.; Ćirić, A.; Stojković, D.; Soković, M. Antioxidant and antimicrobial activity of Cynara cardunculus extracts. Food Chem. 2008, 107, 861-868. [CrossRef]

45. Petropoulos, S.A.; Fernandes, Â.; Dias, M.I.; Vasilakoglou, I.B.; Petrotos, K.; Barros, L.; Ferreira, I.C.F.R. Nutritional value, chemical composition and cytotoxic properties of common purslane (Portulaca oleracea L.) in relation to harvesting stage and plant part. Antioxidants 2019, 8, 293. [CrossRef] [PubMed]

46. Da Silva, L.P.; Pereira, E.; Pires, T.C.S.P.; Alves, M.J.; Pereira, O.R.; Barros, L.; Ferreira, I.C.F.R. Rubus ulmifolius Schott fruits: A detailed study of its nutritional, chemical and bioactive properties. Food Res. Int. 2019, 119, 34-43. [CrossRef] [PubMed]

47. Ferreira-Dias, S.; Gominho, J.; Baptista, I.; Pereira, H. Pattern recognition of cardoon oil from different large-scale field trials. Ind. Crops Prod. 2018, 118, 236-245. [CrossRef]

48. Raccuia, S.A.; Melilli, M.G. Biomass and grain oil yields in Cynara cardunculus L. genotypes grown in a Mediterranean environment. Field Crops Res. 2007, 101, 187-197. [CrossRef] 
49. Maccarone, E.; Fallico, B.; Fanella, F.; Mauromicale, G.; Raccuia, S.A. Possible alternative utilization of Cynara spp. II. Chemical characterization of their grain oil. Ind. Crops Prod. 1999, 10, 229-237. [CrossRef]

50. Chihoub, W.; Dias, M.I.; Barros, L.; Calhelha, R.C.; Alves, M.J.; Harzallah-Skhiri, F.; Ferreira, I.C.F.R. Valorisation of the green waste parts from turnip, radish and wild cardoon: Nutritional value, phenolic profile and bioactivity evaluation. Food Res. Int. 2019, 126, 108651. [CrossRef]

51. Petropoulos, S.A.A.; Karkanis, A.; Martins, N.; Ferreira, I.C.F.R. Edible halophytes of the Mediterranean basin: Potential candidates for novel food products. Trends Food Sci. Technol. 2018, 74, 69-84. [CrossRef]

52. Petropoulos, S.A.; Fernandes, Â.; Dias, M.I.; Pereira, C.; Calhelha, R.C.; Chrysargyris, A.; Tzortzakis, N.; Ivanov, M.; Sokovic, M.D.; Barros, L.; et al. Chemical composition and plant growth of Centaurea raphanina subsp. mixta plants cultivated under saline conditions. Molecules 2020, 25, 2204. [CrossRef] [PubMed]

53. Pereira, C.; Barros, L.; Ferreira, I.C.F.R. Analytical tools used to distinguish chemical profiles of plants widely consumed as infusions and dietary supplements: Artichoke, milk thistle, and borututu. Food Anal. Methods 2014, 7, 1604-1611. [CrossRef]

54. Šušaníková, I.; Balleková, J.; Štefek, M.; Hošek, J.; Mučaji, P. Artichoke leaf extract, as AKR1B1 inhibitor, decreases sorbitol level in the rat eye lenses under high glucose conditions ex vivo. Phyther. Res. 2018, 32, 2389-2395. [CrossRef]

55. Miláčková, I.; Kapustová, K.; Mučaji, P.; Hošek, J. Artichoke Leaf Extract Inhibits AKR1B1 and Reduces NF-кB Activity in Human Leukemic Cells. Phyther. Res. 2017, 31, 488-496. [CrossRef] [PubMed]

56. Hernández-Hernández, O.; Ruiz-Aceituno, L.; Sanz, M.L.; Martínez-Castro, I. Determination of free inositols and other low molecular weight carbohydrates in vegetables. J. Agric. Food Chem. 2011, 59, 2451-2455. [CrossRef] [PubMed]

57. Nicoletto, C.; Santagata, S.; Tosini, F.; Sambo, P. Qualitative and healthy traits of different Italian typical artichoke genotypes. CyTA J. Food 2013, 11, 108-113. [CrossRef]

58. Lombardo, S.; Restuccia, C.; Muratore, G.; Barbagallo, R.N.; Licciardello, F.; Pandino, G.; Scifò, O.; Mazzaglia, A.; Ragonese, F.; Mauromicale, G. Effect of nitrogen fertilization on the overall quality of minimally processed globe artichoke heads. J. Sci. Food Agric. 2017, 97, 650-658. [CrossRef]

59. Di Salvo, R.; Fadda, C.; Sanguinetti, A.M.; Naes, T.; Del Caro, A. Effect of harvest time and geographical area on sensory and instrumental texture profile of a PDO artichoke. Int. J. Food Sci. Technol. 2014, 49, 1231-1237. [CrossRef]

60. Leroy, G.; Grongnet, J.F.; Mabeau, S.; le Corre, D.; Baty-Julien, C. Changes in inulin and soluble sugar concentration in artichokes (Cynara scolymus L.) during storage. J. Sci. Food Agric. 2010, 90, 1203-1209. [CrossRef]

61. Barracosa, P.; Barracosa, M.; Pires, E. Cardoon as a Sustainable Crop for Biomass and Bioactive Compounds Production. Chem. Biodivers. 2019, 16, e1900498. [CrossRef]

62. Vergauwen, R.; Van Laere, A.; Van Den Ende, W. Properties of fructan:fructan 1-fructosyltransferases from chicory and globe thistle, two asteracean plants storing greatly different types of inulin. Plant. Physiol. 2003, 133, 391-401. [CrossRef]

63. Roberfroid, M.B. Introducing inulin-type fructans. Br. J. Nutr. 2005, 93, S13-S25. [CrossRef] [PubMed]

64. Petropoulos, S.A.; Fernandes, Â.; Dias, M.I.; Pereira, C.; Calhelha, R.; Gioia, F.D.; Tzortzakis, N.; Ivanov, M.; Sokovic, M.; Barros, L.; et al. Wild and cultivated Centaurea raphanina subsp. mixta: A valuable source of bioactive compounds. Antioxidants 2020, 9, 314. [CrossRef] [PubMed]

65. Tarasov, D.; Leitch, M.; Fatehi, P. Lignin-carbohydrate complexes: Properties, applications, analyses, and methods of extraction: A review. Biotechnol. Biofuels 2018, 11, 1-28.

(C) 2020 by the authors. Licensee MDPI, Basel, Switzerland. This article is an open access article distributed under the terms and conditions of the Creative Commons Attribution (CC BY) license (http://creativecommons.org/licenses/by/4.0/). 